\title{
Optical IP network system using 16 wavelengths of Coarse WDM technologies and a Layer-3 switch
}

\author{
V. H. Nguyen, ${ }^{* 1}$ A. Marsden, ${ }^{2}$ S. Fujimoto, ${ }^{2}$ and Y. Katsuyama ${ }^{2}$ \\ ${ }^{1}$ School of Computer and Communication, Hunan University, Changsha 410082, China, \\ ${ }^{2}$ Department of Electrical and Electronic Systems, Osaka Prefecture University, Sakai, Osaka, 599-8531, Japan
}

Received June 09, 2013; accepted September 26, 2013; published September 30, 2013

\begin{abstract}
We have proposed and experimentally demonstrated an optical network system using 16 wavelengths of Coarse WavelengthDivision-Multiplexing (CWDM) technology and a Layer-3 Switch. For keeping the speed of $1000 \mathrm{Mbps}$ at the bus IFs, CWDM technology is employed, and 16 wavelengths are allocated to 16 bus IF, respectively. The system consists of a gateway which contains a controller and a server to connect terminals and public providers. The optical IP networks can be scalable by adjusting the number of wavelengths and optical transceiver. We believe this novel scheme is a practical scheme to be applied for future broadband networks.
\end{abstract}

Recently, optical fiber networks are expected to satisfy the increasing traffic demands of the internet [1], by utilizing their high-capacity and low-cost characteristics. The CWDM technology is effective for such an application, because no wavelength control is necessary and the device is low cost. Many approaches have been tried to develop huge traffic volumes through optical IP network architecture [2]. These approaches include not only core backbone networks but also to metropolitan/regional and access networks. The cost reduction is the major issue to be solved when applying an optical IP network. IP-based optical networking is investigated [3] for lots of different applications by many different approaches. Another approach is dark-fiber or customer-owned networks [4]. This new type of network is becoming increasingly common among large enterprise networks, university research network, and government departments in the USA and Canada. In the networks, enterprises, government departments and other organizations acquire optical fibers for their own communication services. A big advantage of customerowned networks is a much lower cost for one-time capital required for the fibers, instead of conventional cost per month for a bandwidth. Thereafter, some increase in the bandwidth only requires a simple equipment upgrade. The IP-over-CWDM networks should be managed and controlled by a centralized control system for the lightpath reconfiguration [5-6]. An IP-over-CWDM ring network, which consists of dual fibers and optical nodes with reconfigurable optical add/drop multiplexers, is a promising candidate for optical IP networks [7-8]. In an optical IP network system, the premises size determines how many rooms or how many telephone services and

\footnotetext{
*E-mail: viet02342000@yahoo.com
}

PCs should be provided, and where and how cables should be constructed. The construction should be variable, depending on the new construction and replacement. In this paper, we have proposed and experimentally demonstrated an optical network system using 16 wavelengths of CWDM technology and a Layer3 Switch to give an integrated broadband.

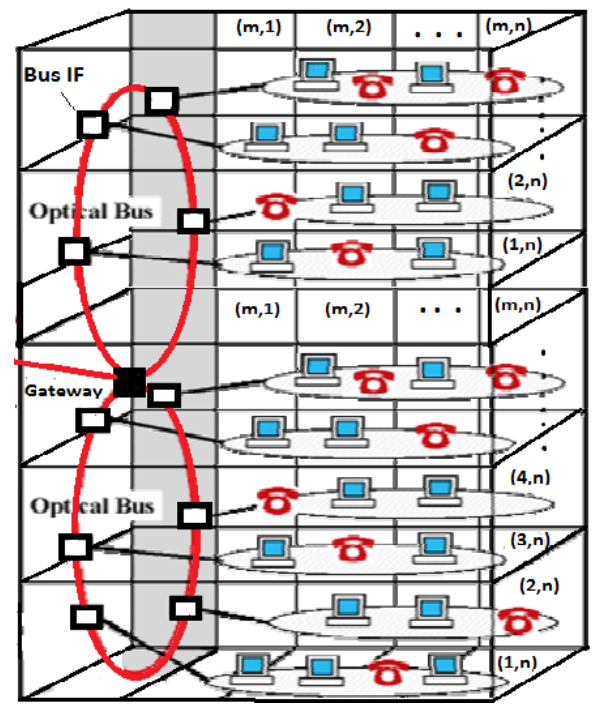

Fig. 1. Optical network system.

In Figure 1, there are $n$ rooms in each floor and $2 m$ floors. We suppose that if each room has one customer, then there are $2 m n$ customers in this building. A single-mode optical fiber (G652) is installed to connect all the floors in half of the building as an optical bus. The maximum optical bus number can be 2 per controller. A gateway which contains a controller and a server is installed in this building, being attached to the optical bus. This gateway has a switching function to connect all the terminals in the building and service provider for managing the communications and processing the administrative tasks. A bus interface (IF) is attached to the optical bus, supplying communication capability to the terminals in the floor. The telephone sets or PCs are connected to the bus IF. The maximum number of bus IFs can be 16 , to be attached to one optical bus. Thus, the maximum number of bus IFs inside the building can be 32. The customer number can range from 8 to 16 per 
optical bus IF, so the maximum number of customers inside this building can be 512 so as they can use high speed internet. The transmission speed is $1000 \mathrm{Mbps}$ for each wavelength. The nominal speed is $100 \mathrm{Mbps} /$ customer, which may be of lesser value, depending on the customer number. The fastest speed is about 93Mbps and the slowest speed is about $56 \mathrm{Mbps} /$ customer, which is obtained by dividing the fastest speed $900 \mathrm{Mbps}$ of the bus interface by the largest customer number per bus IF.

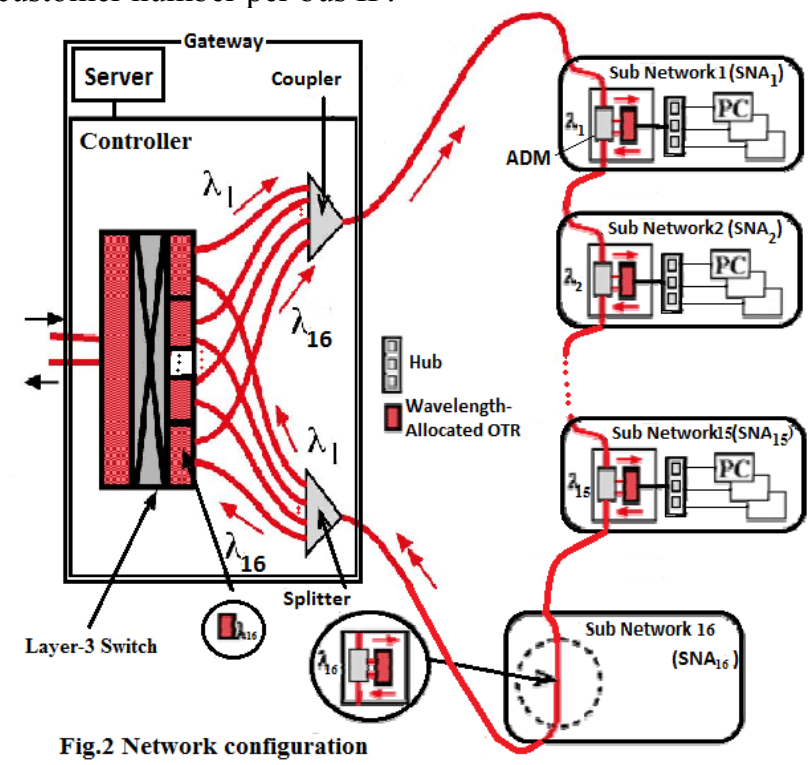

Figure 2 shows the system configuration. For keeping the speed of 1000Mbps at the bus IFs, Coarse WDM technology is employed, and 16 wavelengths are allocated to 16 bus IFs, respectively. The lightwaves from optical transceivers (OTR) with different wavelengths are multiplexed by a coupler in the controller, and transmitted through the optical bus. In the system, electrical/optical hybrid lambda switching was designed. This is based on the combination of WDM in the optical domain and the layer-3 switch in the electrical domain. A particular wavelength is allocated to each floor. As shown in Fig.2, one of the gigabit wavelength-allocated optical transceivers (GbWA-TRs) is operated at a wavelength of $1310 \mathrm{~nm}$-band for a public provider connection, and the others are operated at $1550 \mathrm{~nm}$-band. The layer-3 switch has an IP-based routing function, and is used for routing packets. Each terminal is given an IP address that all the terminals in a floor belong to, there being one subnetwork in that floor, and each floor having a different sub-network. The virtual LANs are specified in the layer3 switch, corresponding to each floor and the public provider. The bus IF consists of an optical add/drop module (ADM) and a GbWA-TR. The bus IFs have a function to give interfaces between terminals belonging to one sub network and the optical bus. The ADMs have a function to add/drop one wavelength, and pass the other wavelengths. The GbWA-TR has an electrical gigabit-T interface, providing a port to a hub connection or a direct connection to a personal computer (PC).

Figure 2 also shows how scalable this network is, by illustrating it with an example of a new sub network added to the existing optical bus. For example, $15 \mathrm{sub}$ networks have already been constructed. When adding the $16^{\text {th }}$ sub network, a new bus IF is employed, inserted to the bus as shown in Fig. 2, and a new OTR is also inserted inside the controller, to which a new wavelength $\lambda_{16}$ was allocated. The bus IF insertion is simply done by connecting the 2 ports of the bus IF and the optical fiber ends of the bus with optical connectors. The OTR can also simply inserted by connecting. The information for the packet routing from/to the new sub network can be added in the routing table and the forwarding database in the layer-3 switch. It is really a simple and low cost solution because no additional fiber installation is required, and no special tools are needed. When removing one of the bus IFs, the procedures are similar to those for the addition.

\begin{tabular}{|c|c|c|c|c|c|c|c|c|c|c|c|}
\hline \multirow{3}{*}{$\begin{array}{l}\text { Wave- } \\
\text { length } \\
(\mathrm{nm})\end{array}$} & \multicolumn{11}{|c|}{ Optical Loss (dB) } \\
\hline & \multirow[t]{2}{*}{ Coupler } & \multicolumn{10}{|c|}{ ADM } \\
\hline & & 1 & 2 & 3 & 4 & 5 & 6 & 7 & 8 & 9 & 10 \\
\hline 1460 & 0.45 & 0.42 & 0.45 & 0.44 & 0.41 & 0.45 & 0.49 & 0.61 & 0.66 & 0. & 0.58 \\
\hline 1470 & 0.47 & 0.47 & 0.48 & 0.42 & 0.4 & 0.45 & 0.48 & 0.75 & 0.86 & 0.7 & 0.65 \\
\hline 1480 & 0.47 & 0.41 & 0.45 & 0.44 & 0.4 & 0.43 & 0.47 & 0.59 & 0.69 & 0.6 & 0.53 \\
\hline 1490 & 0.46 & 0.29 & 0.38 & 0.49 & 0.48 & 0.48 & 0.69 & 0.91 & 0.62 & 0.5 & 0.51 \\
\hline 1500 & 0.8 & 0.45 & 0.48 & 0.52 & 0.51 & 0.5 & 0.53 & 0.63 & 0.58 & 0.5 & 0.69 \\
\hline 1510 & 0.9 & 0.61 & 0.49 & 0.32 & 0.42 & 0.54 & 0.63 & 0.74 & 0.66 & 0.6 & 0.64 \\
\hline 1520 & 0.9 & 0.57 & 0.51 & 0.42 & 0.47 & 0.52 & 0.59 & 0.63 & 0.61 & 0.5 & 0.59 \\
\hline 1530 & 0.89 & 0.51 & 0.47 & 0.32 & 0.42 & 0.55 & 0.62 & 0.67 & 0.53 & 0.4 & \\
\hline 1540 & 1.06 & 0.53 & 0.49 & 0.39 & 0.42 & 0.51 & 0.59 & 0.61 & 0.56 & & \\
\hline 1550 & 1.17 & 0.62 & 0.45 & 0.36 & 0.39 & 0.47 & 0.52 & 0.57 & & & \\
\hline 1560 & 1.21 & 0.59 & 0.53 & 0.45 & 0.49 & 0.51 & 0.53 & & & & \\
\hline 1570 & 1.32 & 0.56 & 0.57 & 0.59 & 0.54 & 0.52 & & & & & \\
\hline 1580 & 1.55 & 0.58 & 0.56 & 0.61 & 0.58 & & & & & & \\
\hline 1590 & 1.72 & 0.59 & 0.51 & 0.41 & & & & & & & \\
\hline 1600 & 2.07 & 0.57 & 0.56 & & & & & & & & \\
\hline 1610 & 2.27 & 0.58 & & & & & & & & & \\
\hline \multirow{3}{*}{$\begin{array}{l}\text { Wave- } \\
\text { length } \\
\text { (nm) }\end{array}$} & \multicolumn{10}{|c|}{ Optical Loss (dB) } & \\
\hline & \multicolumn{8}{|c|}{ ADM } & \multirow[t]{2}{*}{ Total } & & \\
\hline & 11 & 1 & & 13 & 14 & & 15 & 16 & & & \\
\hline 1460 & 0.52 & 0.4 & & 0.63 & 0.65 & & .53 & 0.47 & 10.2 & & 26.12 \\
\hline 1470 & 0.59 & 0.4 & & 0.71 & 0.78 & & .51 & & 10.1 & & 26.23 \\
\hline 1480 & 0.57 & 0.5 & & 0.61 & 0.63 & & & & 9.45 & & 26.68 \\
\hline 1490 & 0.52 & 0.4 & & 0.56 & & & & & 9.12 & & 26.84 \\
\hline 1500 & 0.58 & 0.5 & & & & & & & 8.84 & & 27.25 \\
\hline 1510 & 0.69 & & & & & & & & 8.5. & & 27.45 \\
\hline 1520 & & & & & & & & & 8.21 & & 27.84 \\
\hline 1530 & & & & & & & & & 7.96 & & 28.12 \\
\hline 1540 & & & & & & & & & 7.79 & & 28.36 \\
\hline 1550 & & & & & & & & & 7.55 & & 28.51 \\
\hline 1560 & & & & & & & & & 7.36 & & 28.69 \\
\hline 1570 & & & & & & & & & 7.18 & & 28.81 \\
\hline 1580 & & & & & & & & & 7.02 & & 28.98 \\
\hline 1590 & & & & & & & & & 6.91 & & 29.12 \\
\hline 1600 & & & & & & & & & 6.79 & & 29.26 \\
\hline 1610 & & & & & & & & & 6.69 & & 29.32 \\
\hline
\end{tabular}

Table 1. Measured losses for Downstream paths

16 wavelengths of coarse WDM standard were specified: 1460, 1470, 1480, 1490, 1500, 1510, 1520, $1530,1540,1550,1560,1570,1580,1590,1600$ and $1610 \mathrm{~nm}$. They are allocated to sub networks from 16 to 1 , respectively. The light sources were DFB lasers and were packaged into the transceivers by standardized 1000BaseF technology. The output powers of GbWA-TRs were 
measured and the results showed more than $+2.1 \mathrm{dBm}$. The minimum detectable level was $-20 \mathrm{dBm}$, leading to a dynamic range of $22 \mathrm{~dB}$. The optical losses of the optical component were also measured in the $1550 \mathrm{~nm}$ band. The results are shown in Table 1 for the downstream. The optical losses of the downstream paths from the controller to one of the bus IF are the total losses of the coupler, passing-through losses of the ADMs and dropping loss at the bus IF. In Table 1, the measured losses are listed for 16 wavelengths, corresponding to each sub network, and the total losses are shown, including a margin of $0.5 \mathrm{~dB}$. The values of the total losses of the paths and the dynamic range of OTRs give us the link fiber lengths of the optical bus, assuming that the average losses of fibers are $0.5 \mathrm{~dB} / \mathrm{km}$, including connection and splicing losses. The estimated fibers lengths are also listed in Table 1 . In the same way, the link fiber lengths were obtained for the upstream paths from each bus IF to the controller. The results clarified that longer lengths of optical bus than $26 \mathrm{~km}$ is possible for all the wavelengths. This is the link length enough to transmit packets inside the residential premises.

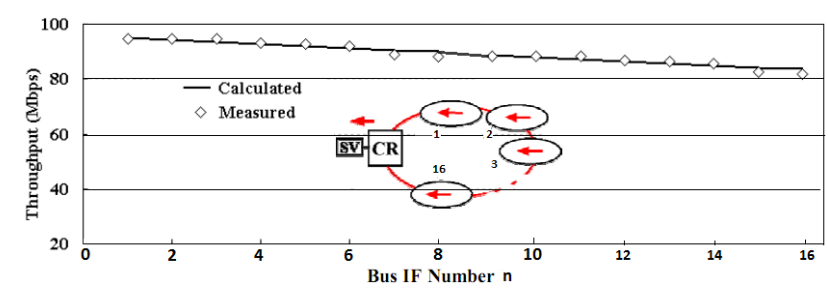

Fig. 3. Measured throughput.

To evaluate more quantitative characteristics of transmission, the throughputs were measured as the limiting performance [9]. The limiting performance was measured as a delay in the network. The delay was measured with a file transfer time measurement tool [9], and the throughput was evaluated as a value of all the data divided by the transfer time. The results are shown in Fig. 3. All the network interface cards of the PCs were 100Base-T and the hub had 100Mbps multi-ports for the PCs and a gigabit port for the bus IF. The network interface card of the server was 1000 Base-T, which was connected to the optical transceiver operated at $1310 \mathrm{~nm}$. Firstly, the throughput was measured, when only one PC in floor 1 sent packets to the server. The throughput was more than $93 \mathrm{Mbps}$, as shown by the plot corresponding to $n=1$ in Fig. 3 . This is a reasonable result, because the data packets were sent together with the overhead in the TCP/IP transmission. Next, the throughputs were measured, when 2 PCs sent packets simultaneously, and the result is shown by a plot for $n=2$. In the same way the throughputs were measured with increasing $n$. The file sizes were $20 \mathrm{MB}$ in all the measurements. When $n$ is equal to or larger than 2, they are the WDM transmission in the optical bus, expecting as same throughput as that by a single PC. However, all the packets are concentrated and controlled in the switch in an electrical domain. This causes a slightly decreasing of the throughput. When the files to be sent have the same size, the throughput $t p(n)$ is given [9] by

$$
\operatorname{tp}(n)=\frac{u_{d}}{t_{u}+n z}
$$

where $u_{d}, t_{u}$, and $z$ are the unit packet size, unit transfer time of one packet and time which one packet passes through the switch, respectively. The value of $u_{d}$ is 1460 byte for IEEE802.3 LAN, and the other values were determined [9] by experiments: $t_{u}=0.122 \mathrm{~ms}$ and $\mathrm{z}=0.001 \mathrm{~ms}$. The calculated values by Eq. (1) are also shown in Fig. 3. When $n=16$ means the throughput when 16 PCs (one PC in each sub network) stared sending packets simultaneously. It is found that the measured and calculated values are in good agreement. This verifies the fact that any client can communicate independently with the public provider with a nominal speed of $88 \mathrm{Mbps}$.

A scalable and reconfigurable 16 wavelengths optical IP network system for residential premises was investigated and constructed. The system consists of a gateway, an optical fiber bus and bus IFs attached to the bus. To evaluate the characteristics of transmission, the throughputs were measured as the limiting performance. The results verify the throughput around $88 \mathrm{Mbps}$ for the bus IF number of up to 16 , indicating the nominal speed of 100 base Ethernet. When measuring the throughput, the bus IF number was increased one by one, indicating that the bus IFs can be added afterwards, thus the network is scalable. We believe this network is a practical scheme which can be applied in a future broadband network.

\section{References}

[1] A.A.M. Saleh, J.M. Simmons, J. Lightwave Tech. 17, 2431 (1999).

[2] J. De Clercq, O. Paridaens, IEEE Comm. Magazines 40, 151 (2002).

[3] A. Jourdan, D. Chiaroni, E. Dotaro, IEEE Comm. Magazines 39, 136 (2001).

[4] B.St Arnaud, J. Wu, B. Kalali, J. Lightwave Tech. 21, 2804 (2003).

[5] M. Hashimoto, A Ueno, M Taniue, S Kawase, O Koyama, Y. Katsuyama, The $9^{\text {th }}$ International Conference on Telecom., Zagreb, Croatia, June 2007.

[6] M. Hashimoto, A. Ueno, M. Taniue, S. Kawase, O. Koyama, Y. Katsuyama, International J. Innovative Comp., Inform. Control 4(6), 1299 (2008).

[7] M. Hashimoto, O. Koyama, M.D. Nooruzzaman, Y. Katsuyama, IEEE Trans. Electrical and Electronic Eng. 4(3), 425 (2009).

[8] M.D. Nooruzzaman, Y. Harada, O. Koyama, Y. Katsuyama, IEICE Trans. Commun. E91-B(10), 3330 (2008).

[9] S. Fujimoto, K. Tsujimura, S. Hayashi, J. Wang and Y. Katsuyama, in Proc. of 7th APCC, 9-12, Tokyo (2001). 